ISSN 1855-3966 (printed edn.), ISSN 1855-3974 (electronic edn.)

ARS MATHEMATICA CONTEMPORANEA 19 (2020) 61-76

https://doi.org/10.26493/1855-3974.2106.423

(Also available at http://amc-journal.eu)

\title{
Dominating sets in finite generalized quadrangles*
}

\author{
Tamás Héger ${ }^{\dagger}$ \\ MTA-ELTE Geometric and Algebraic Combinatorics Research Group, ELTE Eötvös \\ Loránd University, Pázmány Péter sétány 1/C, Budapest, Hungary, H-1117 \\ Lisa Hernandez Lucas \\ Department of Mathematics, Vrije Universiteit Brussel, \\ Pleinlaan 2, 1050 Brussels, Belgium
}

Received 30 August 2019, accepted 25 March 2020, published online 2 November 2020

\begin{abstract}
A dominating set in a graph is a set of vertices such that each vertex not in the set has a neighbor in the set. The domination number is the smallest size of a dominating set. We consider this problem in the incidence graph of a generalized quadrangle. We show that the domination number of a generalized quadrangle with parameters $s$ and $t$ is at most $2 s t+1$, and we prove that this bound is sharp if $s=t$ or if $s=q-1$ and $t=q+1$. Moreover, we give a complete classification of smallest dominating sets in generalized quadrangles where $s=t$, and give some general results for small dominating sets in the general case.

Keywords: Dominating set, finite generalized quadrangle.
\end{abstract}

Math. Subj. Class. (2020): 05B25, 05C69, 51E12

\section{Preliminaries}

Dominating sets in graphs have already been studied in 1958, but there was a boost of interest after the publishing of a survey paper in the '70s by Cockayne and Hedetniemi [3], in which the authors show that the domination problem is related to the well-known problem of colorings of graphs. In [8] a dominating set is defined as follows:

* The authors gratefully acknowledge the support of the FWO-HAS mobility grant 'Substructures in finite projective spaces: algebraic and extremal questions'. The authors also wish to thank Jan De Beule and Leo Storme for the discussions during this work, and the anonymous referee for a clarifying comment.

† Supported by the János Bolyai Research Scholarship of the Hungarian Academy of Sciences and by NKFIH OTKA Grant No. K124950.

E-mail addresses: heger@ caesar.elte.hu (Tamás Héger), lihernan@vub.be (Lisa Hernandez Lucas)

() (i) This work is licensed under https://creativecommons.org/licenses/by/4.0/ 
Definition 1.1. Let $G=(V, E)$ be a graph. The closed neighborhood $N[S]$ of a set of vertices $S$ is defined as the set of vertices adjacent to any vertex in $S$, joint with the vertices of $S$ itself. A set $D \subseteq V$ is a dominating set if $N[D]=V$.

It is desirable to find the smallest dominating sets in a graph. The number of vertices in the smallest dominating set in a graph $G$ is the domination number of $G$, and a common notation for it is $\Gamma(G)$.

The problem of domination has been studied before in incidence graphs of geometric structures, see for instance [6] and [11]. Also, perfect dominating sets of the incidence graphs of finite generalized quadrangles were considered in [4] (see also [9]), and for the particular quadrangle $Q(4, q)$, they were studied in detail in [2]; see Section 5 for further information. In this paper, we will consider dominating sets in the incidence graph of finite generalized quadrangles.

Generalized quadrangles were first introduced by Tits [14]. In [13], Payne and Thas give the following definition of finite generalized quadrangles:

Definition 1.2. A finite generalized quadrangle $\operatorname{GQ}(s, t)$ with parameters $s$ and $t$, where $s, t \geq 1$, is a point-line incidence structure $(\mathcal{P}, \mathcal{B}, \mathrm{I})$, in which $\mathcal{P}$ is the set of points, $\mathcal{B}$ is the set of lines and $\mathrm{I}$ is a symmetric point-line incidence relation, satisfying the following axioms:

- Each point is incident with $t+1$ lines and two distinct points are incident with at most one line.

- Each line is incident with $s+1$ points and two distinct lines are incident with at most one point.

- If $x$ is a point and $L$ is a line not incident with $x$, then there is a unique pair $(y, M) \in$ $\mathcal{P} \times \mathcal{B}$ for which $x$ I $M$ I $y$ I $L$.

We will refer to this third property as the projection property. A generalized quadrangle $\operatorname{GQ}(s, t)$ with parameters $s$ and $t$ is said to have $\operatorname{order}(s, t)$. It is well known that the number of points in a generalized quadrangle of order $(s, t)$ is $(s+1)(s t+1)$, and the number of lines is $(t+1)(s t+1)$. For two points $P$ and $Q$, we will write $P \sim Q$ if there exists a line incident with both (that is, they are collinear), and we will use this notation dually for lines as well.

For readability, we will refer to a dominating set in the incidence graph of $\operatorname{GQ}(s, t)$ and the domination number of the incidence graph of $\mathrm{GQ}(s, t)$ as a dominating set in $\mathrm{GQ}(s, t)$ and the domination number of $\mathrm{GQ}(s, t)$.

When viewed from a geometric perspective, a dominating set in $\operatorname{GQ}(s, t)$ becomes the union of a set of points and a set of lines such that each point which is not in the set is incident with a line from the set and such that each line which is not in the set is incident with a point from the set. This is closely related to the concept of blocking sets and the dual concept of covers.

Definition 1.3. A blocking set in $\operatorname{GQ}(s, t)$ is a set of points such that each line is incident with at least one of these points. A cover in $\operatorname{GQ}(s, t)$ is a set of lines such that each point is incident with at least one of these lines.

A blocking set $\mathcal{O}$ such that no two points from $\mathcal{O}$ are collinear is called an ovoid, a cover $\mathcal{S}$ such that no two lines from $\mathcal{S}$ are concurrent is called a spread. An arbitrary set 
of points $\mathcal{O}$ such that no two points from the set are collinear is called a partial ovoid, an arbitrary set of lines $\mathcal{S}$ such that no two lines from $\mathcal{S}$ are concurrent is called a partial spread. Let us note that an ovoid or a spread in $\operatorname{GQ}(s, t)$ contains exactly $s t+1$ elements, and this is the smallest possibe size for a blocking set or a covering set.

In Section 2 we will show that there exist dominating sets in $\operatorname{GQ}(s, t)$ of size $2 s t+1$ and that the union of a blocking set and a cover exceeds this size. This motivates us to call a dominating set in GQ $(s, t)$ small when it has size at most $2 s t+1$. This section also lists some properties of small dominating sets in $\operatorname{GQ}(s, t)$. In Section 3 we show that all small dominating sets in $\operatorname{GQ}(s, t)$, where $|s-t| \leq 3$ must have size $2 s t+1$, which shows that the domination number in this case is $2 s t+1$. In particular, this gives us the domination number of $\operatorname{GQ}(q, q)$ and $\operatorname{GQ}(q-1, q+1)$. In Section 4 we give a classification of small dominating sets in $\operatorname{GQ}(q, q)$. In Section 5 we give a summary of the main results, and add some open problems.

\section{Examples and properties of small dominating sets in $\mathrm{GQ}(s, t)$}

Consider a generalized quadrangle $\mathrm{GQ}(s, t)$. We will construct a dominating set $D$ of size $2 s t+1$ as follows. Let $P$ be a point in $\operatorname{GQ}(s, t)$. Number the lines through $P$ as $\ell_{1}, \ell_{2}, \ldots, \ell_{t+1}$. Now define $\mathcal{P}$ as the set of all points which are incident with one of the first $t$ lines $\ell_{1}, \ldots, \ell_{t}$ through $P$, including $P$ itself. Then $|\mathcal{P}|=s t+1$. Now define $\mathcal{L}$ as the set of lines which intersect the last line $\ell_{t+1}$ in a point different from $P$. Then $|\mathcal{L}|=s t$. Define $D=\mathcal{P} \cup \mathcal{L}$. The construction is also shown in Figure 1 .

The size of $D$ is $2 s t+1$. Now take an arbitrary point $Q$ in $\operatorname{GQ}(s, t)$. If $Q$ is incident with $\ell_{t+1}$, then it is either contained in the dominating set (if $Q=P$ ), or covered by $t$ different lines from the dominating set. So assume $Q$ is not incident with $\ell_{t+1}$. Then by the projection property of generalized quadrangles, there exists a unique point-line pair $(R, m)$ such that $R$ is incident with $\ell_{t+1}$ and $m$ is incident with both $Q$ and $R$. If $m$ is one of the lines $\ell_{1}, \ldots, \ell_{t}$, then $Q$ is a point of the dominating set. Otherwise, $m$ must be a line intersecting $\ell_{t+1}$ in a point different from $P$. Then $m$ is in the dominating set and $Q$ is covered.

Now take an arbitrary line $\ell$. Assume $\ell$ is not incident with $P$. Then again by the projection property of generalized quadrangles, there exists a unique point-line pair $(R, m)$ such that $R$ is incident with $\ell$ and $m$ is incident with both $P$ and $R$. If $R$ is incident with $\ell_{t+1}$, then $\ell$ intersects $\ell_{t+1}$ in a point different from $P$, so it must be one of the lines in the dominating set. Otherwise, $R$ is incident with one of the lines $\ell_{1}, \ldots, \ell_{t}$, hence it is in the dominating set. In this case $\ell$ is blocked.

So $D$ is indeed a dominating set of $\operatorname{GQ}(s, t)$. We now have Theorem 2.1.

Theorem 2.1. For any finite generalized quadrangle $\mathrm{GQ}(s, t)$ there exists a dominating set of size 2 st +1 .

Note that the construction in Theorem 2.1 can be dualized, giving us a second example of a dominating set of size $2 s t+1$. We can also get this dual structure by omitting the point $P$ from the dominating set and adding the line $\ell_{t+1}$ to it. See also Figure 1 .

From a graph-theoretical point of view, we can get the dominating set from Theorem 2.1 or its dual as follows. Fix one edge $\{P, \ell\}$ in the incidence graph, then all points with distance two from $\{P, \ell\}$ together with $\{P\}$ (resp. together with $\{\ell\}$ ) form the dominating 

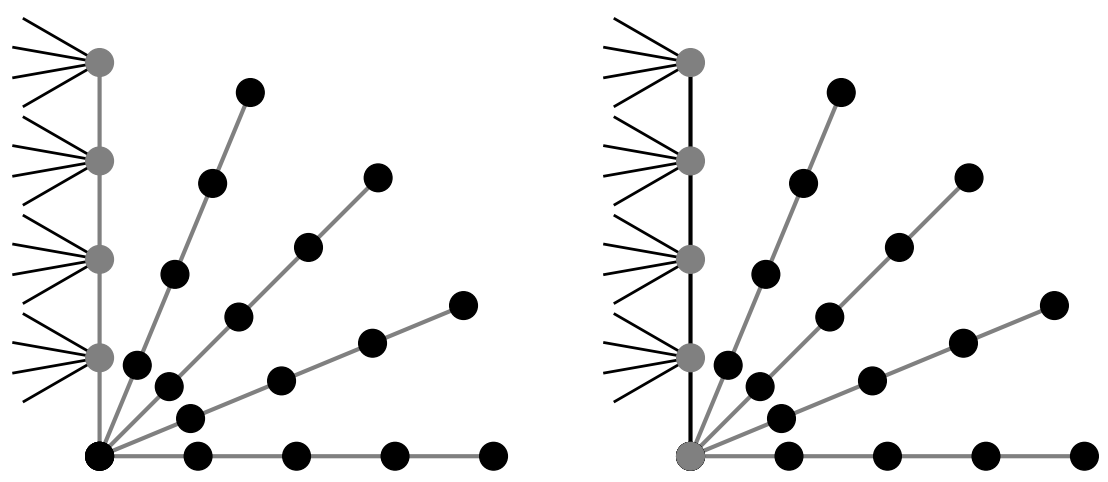

Figure 1: The dominating set from Theorem 2.1 (left) and its dual (right).

set from Theorem 2.1 (resp. its dual) ${ }^{1}$.

Let us note that this dominating set is a maximal independent set in the incidence graph. Maximal independent sets are clearly dominating sets; however, the converse is not always true. Even when the dominating set has the smallest size possible, it is not necessarily a independent set. Families of graphs for which the smallest dominating sets are independent also form a subject of study.

In the case of non-thick generalized quadrangles; that is, when $s=1$ or $t=1$, we immediately have the following result.

Theorem 2.2. The domination number of $\operatorname{GQ}(q, 1)$ and $\mathrm{GQ}(1, q)$ is $2 q+1$; furthermore, dominating sets of size $2 q+1$ are independent.

Proof. By duality, it is sufficient to show this result for $\operatorname{GQ}(q, 1)$. The points and lines of $\mathrm{GQ}(q, 1)$ may naturally be viewed as the $(q+1)^{2}$ points together with the $q+1$ horizontal and $q+1$ vertical lines of a $(q+1) \times(q+1)$ grid.

Let $D$ be a dominating set of size $|D| \leq 2 q+1$, and let $l_{v}$ and $l_{h}$ stand for the number of vertical and horizontal lines in $D$, respectively. If $l_{h}=q+1$, then for each vertical line we must have either the line or a point on the line in $D$, hence $|D| \geq 2 q+2$, a contradiction. Thus $l_{h} \leq q$, and similarly $l_{v} \leq q$.

If $l_{h}=q$, then for all the $q+1$ points on the horizontal line not in $D, D$ must contain either the point or the vertical line through it, whence $|D| \geq 2 q+1$. Since we assumed $|D| \leq 2 q+1$, we now have $|D|=2 q+1$. Moreover, note that $D$ is independent in this case. A similar argument works for $l_{v}=q$.

Suppose now $l_{h} \leq q-1$ and $l_{v} \leq q-1$. The lines of $D$ leave exactly $\left(q+1-l_{h}\right)(q+$ $1-l_{v}$ ) points not covered, which all must be in $D$, whence

$$
(q+1)^{2}-\left(l_{h}+l_{v}\right)(q+1)+l_{h} l_{v}+\left(l_{h}+l_{v}\right) \leq|D| \leq 2 q+1 .
$$

As $l_{h} \leq q-1$ and $l_{v} \leq q-1$, we have $l_{v}+l_{h} \leq 2 q-2$ and $l_{h} l_{v} \geq(q-1)\left(l_{h}+l_{v}-(q-1)\right)$, hence

$2 q+1 \geq(q+1)^{2}-\left(l_{h}+l_{v}\right) q+l_{h} l_{v} \geq(q+1)^{2}-\left(l_{h}+l_{v}\right)-(q-1)^{2} \geq 4 q-(2 q-2)$,

\footnotetext{
${ }^{1}$ The authors wish to thank Sam Mattheus (VUB) for this remark.
} 
a contradiction.

We can conclude that the size of a dominating set $D$ is at least $2 q+1$, and that $D$ is independent in this case. Theorem 2.1 assures that such a dominating set indeed exists.

From now on, we will assume that our generalized quadrangle is thick; that is, $s \geq 2$ and $t \geq 2$.

The most trivial dominating sets are the union of a blocking set and a cover. If an ovoid and a spread exist, then their union forms a dominating set of size $2 s t+2$. So by Theorem 2.1, the union of an ovoid and a spread is definitely not the smallest dominating set. Moreover, we can prove that any dominating set containing a blocking set or a cover exceeds the size $2 s t+1$.

Lemma 2.3. Let $D=\mathcal{P}_{D} \cup \mathcal{L}_{D}$ be a dominating set in $\mathrm{GQ}(s, t), s, t \geq 2$, with $\mathcal{P}_{D}$ the points and $\mathcal{L}_{D}$ the lines of $D$. If $\mathcal{P}_{D}$ is a blocking set or $\mathcal{L}_{D}$ is a cover, then $|D| \geq 2$ st +2 . Moreover, if equality holds, then $\mathcal{P}_{D}$ is an ovoid and $\mathcal{L}_{D}$ is a spread.

Proof. Assume without loss of generality that $\mathcal{P}_{D}$ is a blocking set. The case where $\mathcal{L}_{D}$ is a cover can be showed analogously. Assume $|D| \leq 2 s t+2$, then $\left|\mathcal{P}_{D}\right| \leq 2 s t+2-\left|\mathcal{L}_{D}\right|$. Any point that is not in $\mathcal{P}_{D}$ needs to be covered at least once by a line of $\mathcal{L}_{D}$. But since $\mathcal{P}_{D}$ is a blocking set, each line of $\mathcal{L}_{D}$ contains at least one point of $\mathcal{P}_{D}$, meaning it can only cover at most $s$ points not in $\mathcal{P}_{D}$. This gives us the following inequality:

$$
(s+1)(s t+1) \leq\left|\mathcal{P}_{D}\right|+s\left|\mathcal{L}_{D}\right| \leq 2 s t+2-\left|\mathcal{L}_{D}\right|+s\left|\mathcal{L}_{D}\right|,
$$

whence $(s-1)(s t+1) \leq(s-1)\left|\mathcal{L}_{D}\right|$, and thus $s t+1 \leq\left|\mathcal{L}_{D}\right|$ follows. Since $\mathcal{P}_{D}$ is a blocking set, we have that $\left|\mathcal{P}_{D}\right| \geq s t+1$. But then $|D|=|\mathcal{P}|+|\mathcal{L}| \geq 2 s t+2$, so the lower bound on $|D|$ is proved.

Assume now that equality holds. Then $\left|\mathcal{P}_{D}\right|=\left|\mathcal{L}_{D}\right|=s t+1$ and $\mathcal{L}_{D}$ covers each point not in $D$ exactly once. We want to show that $\mathcal{L}_{D}$ covers each point of $\mathcal{P}_{D}$ exactly once as well. As $\mathcal{P}_{D}$ is a blocking set, its size implies it being an ovoid, so each line of $\mathcal{L}_{D}$ covers at most one point of $\mathcal{P}_{D}$. Suppose that there exists a point $P \in \mathcal{P}_{D}$ not covered by $\mathcal{L}_{D}$. Then each of the $(t+1) s$ points collinear with $P$, which are not in $\mathcal{P}_{D}$, must be covered by a line of $\mathcal{L}_{D}$ which, due to the projection property, are pairwise distinct, implying $\left|\mathcal{L}_{D}\right| \geq s t+s>s t+1$, a contradiction. Hence $\mathcal{L}_{D}$ is a cover of size $s t+1$, that is, a spread.

Lemma 2.3, together with Theorem 2.1, motivates the following definition:

Definition 2.4. Let $D$ be a dominating set in $\operatorname{GQ}(s, t)$. Then $D$ is a small dominating set if $|D| \leq 2 s t+1$.

The following two lemmas also provide us with some information regarding the size of a dominating set or, more precisely, regarding the size of the set of points and the set of lines contained in a dominating set.

Lemma 2.5. Let $\mathcal{P}$ be an arbitrary point set in $\mathrm{GQ}(s, t)$. Assume there exists a number $\Delta$ such that

$$
\forall P \in \mathcal{P}:|\{Q \in \mathcal{P} \mid Q \neq P, Q \sim P\}| \geq \Delta .
$$

Then the number of lines in $\mathrm{GQ}(s, t)$ not blocked by $\mathcal{P}$ is at least

$$
(t+1)(s t+1-|\mathcal{P}|)+\frac{|\mathcal{P}| \Delta}{s+1} .
$$


Proof. For any line $\ell$ in $\mathrm{GQ}(s, t)$, define the degree $\mathrm{d}(\ell)$ of $\ell$ as the number of points in $\mathcal{P}$ which are incident with $\ell$. Let $\mathcal{L}_{b}$ be the set of lines blocked by $\mathcal{P}$. Then

$$
\left|\mathcal{L}_{b}\right|=|\mathcal{P}|(t+1)-\sum_{\ell \in \mathcal{L}_{b}}(\mathrm{~d}(\ell)-1) .
$$

Now let $X:=\{(P, Q) \mid P, Q \in \mathcal{P}, P \neq Q, P \sim Q\}$. Then we have the following inequalities:

$$
|\mathcal{P}| \Delta \leq|X|=\sum_{\ell \in \mathcal{L}_{b}} \mathrm{~d}(\ell)(\mathrm{d}(\ell)-1) \leq(s+1) \sum_{\ell \in \mathcal{L}_{b}}(\mathrm{~d}(\ell)-1),
$$

implying that $\frac{|\mathcal{P}| \Delta}{s+1} \leq \sum_{\ell \in \mathcal{L}_{b}}(\mathrm{~d}(\ell)-1)$. Together with (2.1) this yields:

$$
\left|\mathcal{L}_{b}\right| \leq|\mathcal{P}|(t+1)-\frac{|\mathcal{P}| \Delta}{s+1} .
$$

Since the total number of lines in $\mathrm{GQ}(s, t)$ is $(t+1)(s t+1)$ it follows that the number of lines not blocked by $\mathcal{P}$ is at least $(t+1)(s t+1-|\mathcal{P}|)+\frac{|\mathcal{P}| \Delta}{s+1}$.

If $\mathcal{P}$ is the point set of a dominating set, then Lemma 2.5 gives a lower bound on the number of lines contained in this dominating set. By dualizing this lemma we find:

Lemma 2.6. Let $\mathcal{L}$ be an arbitrary line set in $\mathrm{GQ}(s, t)$. Assume there exists a number $\Delta$ such that

$$
\forall \ell \in \mathcal{L}:|\{m \in \mathcal{L} \mid m \neq \ell, m \sim \ell\}| \geq \Delta .
$$

Then the number of points in $\mathrm{GQ}(s, t)$ not covered by the line set $\mathcal{L}$ is at least

$$
(s+1)(s t+1-|\mathcal{L}|)+\frac{|\mathcal{L}| \Delta}{t+1} .
$$

Notation 2.7. Let $D$ be a dominating set in $\operatorname{GQ}(s, t)$. Let $\mathcal{P}_{D}$ and $\mathcal{L}_{D}$ denote the point set and the line set of $D$, resp. Define $\mathcal{P}^{\prime}$ and $\mathcal{L}^{\prime}$ as the set of points and the set of lines resp., that are not covered by $\mathcal{L}_{D}$ and not blocked by $\mathcal{P}_{D}$ resp. We will use this notation in the sequel implicitly. Note that by the definition of a dominating set, $\mathcal{P}^{\prime} \subseteq \mathcal{P}_{D}$ and $\mathcal{L}^{\prime} \subseteq \mathcal{L}_{D}$.

The following Lemma allows us to apply Lemma 2.5.

Lemma 2.8. Let $D$ be a dominating set in $\operatorname{GQ}(s, t)$. For any point in $\mathcal{P}^{\prime}$, the number of points in $\mathcal{P}^{\prime}$ collinear with it is at least $\Delta_{\mathcal{P}}:=s t+s-\left|\mathcal{L}_{D}\right|$. For any line in $\mathcal{L}^{\prime}$ the number of lines in $\mathcal{L}^{\prime}$ concurrent with it is at least $\Delta_{\mathcal{L}}:=s t+t-\left|\mathcal{P}_{D}\right|$.

Proof. Let $P$ be an arbitrary point in $\mathcal{P}^{\prime}$. Then each line of $D$ covers at most one point collinear with $P$. Hence, there are at least $\Delta_{\mathcal{P}}:=(t+1) s-\left|\mathcal{L}_{D}\right|$ points collinear with $P$ which are not covered by a line of $D$. These points must be in $\mathcal{P}^{\prime}$. So each point of $\mathcal{P}^{\prime}$ is collinear with at least $\Delta_{\mathcal{P}}$ other points of $\mathcal{P}^{\prime}$. Dually, we have that each line of $\mathcal{L}^{\prime}$ is concurrent with at least $\Delta_{\mathcal{L}}:=s t+t-\left|\mathcal{P}_{D}\right|$ other lines of $\mathcal{L}^{\prime}$.

From the next lemma follows that $\Delta_{\mathcal{P}}$ and $\Delta_{\mathcal{L}}$ are non-negative. 
Lemma 2.9. Let $D$ be a dominating set in $\mathrm{GQ}(s, t)$. If $|D| \leq 2 s t+1$, then

$$
\begin{aligned}
& s t-s+1 \leq\left|\mathcal{P}_{D}\right| \leq s t+t, \\
& s t-t+1 \leq\left|\mathcal{L}_{D}\right| \leq s t+s .
\end{aligned}
$$

Proof. Assume $\left|\mathcal{P}_{D}\right|=s t+1-\epsilon$, then at least $\epsilon(t+1)$ lines are not blocked by $\mathcal{P}_{D}$ and have to be in $\mathcal{L}_{D}$. This implies that

$$
2 s t+1 \geq|D|=\left|\mathcal{P}_{D}\right|+\left|\mathcal{L}_{D}\right| \geq s t+1-\epsilon+\epsilon(t+1),
$$

from which follows that $\epsilon \leq s$. Hence $\left|\mathcal{P}_{D}\right| \geq s t-s+1$. From this we obtain

$$
2 s t+1 \geq|D|=\left|\mathcal{P}_{D}\right|+\left|\mathcal{L}_{D}\right| \geq s t-s+1+\left|\mathcal{L}_{D}\right|,
$$

hence $\left|\mathcal{L}_{D}\right| \leq s t+s$. The other two inequalities follow similarly.

\section{The domination number of $\operatorname{GQ}(s, t),|s-t|$ small}

Theorem 3.1. The domination number of $\mathrm{GQ}(s, t)$, where $|s-t| \leq 3$, is $2 s t+1$.

Proof. By Theorem 2.1, it is enough to show $\Gamma(\mathrm{GQ}(s, t)) \geq 2 s t+1$. Assume a dominating set $D$ exists with size smaller than $2 s t+1$. When lines or points are added to a dominating set, it still remains a dominating set, so without loss of generality we may assume that $D$ has size $|D|=2 s t$.

Let $l=\left|\mathcal{L}_{D}\right|$ and $p=\left|\mathcal{P}_{D}\right|$, and let $\Delta_{\mathcal{P}}$ and $\Delta_{\mathcal{L}}$ be as in Lemma 2.8. By Lemma 2.5 we have that the number of lines not blocked by $\mathcal{P}^{\prime}$ is at least $\left(s t+1-\left|\mathcal{P}^{\prime}\right|\right)(t+1)+\frac{\left|\mathcal{P}^{\prime}\right| \Delta_{\mathcal{P}}}{s+1}$. Since each point of $D$ can block at most $t+1$ lines, the number of lines $\left|\mathcal{L}^{\prime}\right|$ not blocked by $D$ is at least

$$
\begin{aligned}
\left|\mathcal{L}^{\prime}\right| & \geq\left(s t+1-\left|\mathcal{P}^{\prime}\right|\right)(t+1)+\frac{\left|\mathcal{P}^{\prime}\right| \Delta_{\mathcal{P}}}{s+1}-\left(p-\left|\mathcal{P}^{\prime}\right|\right)(t+1) \\
& =(s t+1-p)(t+1)+\frac{\left|\mathcal{P}^{\prime}\right| \Delta_{\mathcal{P}}}{s+1} .
\end{aligned}
$$

Dually, by Lemma 2.6, we find that

$$
\left|\mathcal{P}^{\prime}\right| \geq(s t+1-l)(s+1)+\frac{\left|\mathcal{L}^{\prime}\right| \Delta_{\mathcal{L}}}{t+1} .
$$

Suppose, say, $l \leq p$ (we may consider the dual quadrangle otherwise). Let $0 \leq \epsilon \leq t$ be such that $p=s t+\epsilon, l=s t-\epsilon$ (cf. Lemma 2.9). Filling in these and $\Delta_{\mathcal{P}}=s t+s-l=s+\varepsilon$ and $\Delta_{\mathcal{L}}=s t+t-p=t-\varepsilon$, multiplying by $s+1$ and $t+1$ resp., and rearranging we get

$$
\begin{gathered}
(s+1)\left|\mathcal{L}^{\prime}\right|-(s+\varepsilon)\left|\mathcal{P}^{\prime}\right| \geq(1-\epsilon)(t+1)(s+1), \\
(t+1)\left|\mathcal{P}^{\prime}\right|-(t-\varepsilon)\left|\mathcal{L}^{\prime}\right| \geq(1+\epsilon)(t+1)(s+1) .
\end{gathered}
$$

Suppose $\left|\mathcal{P}^{\prime}\right| \leq\left|\mathcal{L}^{\prime}\right|$. Then (3.2) yields

$$
(1+\epsilon)(t+1)(s+1) \leq(t+1)\left|\mathcal{P}^{\prime}\right|-(t-\varepsilon)\left|\mathcal{L}^{\prime}\right| \leq(1+\epsilon)\left|\mathcal{L}^{\prime}\right| \leq(1+\epsilon)\left|\mathcal{L}_{D}\right| \leq(1+\epsilon) s t,
$$

a contradiction. Hence $\left|\mathcal{P}^{\prime}\right|>\left|\mathcal{L}^{\prime}\right|$. Then (3.1) gives

$$
(1-\epsilon)(t+1)(s+1) \leq(s+1)\left|\mathcal{L}^{\prime}\right|-(s+\varepsilon)\left|\mathcal{P}^{\prime}\right|<(1-\epsilon)\left|\mathcal{P}^{\prime}\right| \leq(1-\epsilon)(s t+t),
$$


a contradiction if $\epsilon \leq 1$; thus $\epsilon \geq 2$. Adding up (3.1) and (3.2) we find

$2(t+1)(s+1) \leq(t-s-\epsilon+1)\left|\mathcal{P}^{\prime}\right|-(t-s-\epsilon-1)\left|\mathcal{L}^{\prime}\right|=(t-s-\epsilon-1)\left(\left|\mathcal{P}^{\prime}\right|-\left|\mathcal{L}^{\prime}\right|\right)+2\left|\mathcal{P}^{\prime}\right|$.

As $t-s \leq 3, \epsilon+1 \geq 3,\left|\mathcal{P}^{\prime}\right|-\left|\mathcal{L}^{\prime}\right|>0$ and $\left|\mathcal{P}^{\prime}\right| \leq\left|\mathcal{P}_{D}\right| \leq s t+t$, this is a contradiction. Consequently, $|D|>2 s t$.

Corollary 3.2. The domination number of $\mathrm{GQ}(q, q)$ is $2 q^{2}+1$, and the domination number of $\mathrm{GQ}(q+1, q-1)$ and $\mathrm{GQ}(q-1, q+1)$ is $2 q^{2}-1$.

This corollary applies to the well-known quadrangles $W(q), Q(4, q), T_{2}(O)$ (these have order $(q, q)), T_{2}^{*}(O)$ (of order $(q-1, q+1), q$ even), $\operatorname{AS}(q)$ (of order $(q-1, q+1)$, $q$ odd) and their duals (of order $(q+1, q-1)$ ). Let us note that these quadrangles yield isomorphic incidence graphs in many cases. Clearly, the incidence graphs of a GQ and its dual are isomorphic. Let us now fix $q$. It is known that $W(q)$ is isomorphic to the dual of $Q(4, q)$, and that $T_{2}(O)$ is isomorphic to $Q(4, q)$ if and only if the oval $O$ is a conic [13, Section 3.2], which is certainly the case when $q$ is odd by B. Segre's celebrated result. However, when $q$ is even, $O$ may be an oval that is not a conic, in which case the construction $T_{2}(O)$ gives new instances of GQs of order $(q, q)$ and corresponding incidence graphs. In case of order $(q-1, q+1), q$ even, there are also examples of GQs other than $T_{2}^{*}(O)[13]$.

\section{Classification of the smallest dominating sets in $\operatorname{GQ}(q, q)$}

Corollary 3.2 shows that all small dominating sets in $\operatorname{GQ}(q, q)$ have size $2 q^{2}+1$. Moreover, we already have two constructions of small dominating sets, namely the construction from Theorem 2.1 and its dual. In this section we show that these are the only two small dominating sets.

First we need a few lemmas regarding the structure of small dominating sets in $\operatorname{GQ}(q, q)$.

Lemma 4.1. Let $D=\mathcal{P}_{D} \cup \mathcal{L}_{D}$ be a dominating set in $\mathrm{GQ}(q, q)$ of size $2 q^{2}+1$. Then $\mathcal{P}^{\prime}$ is not a partial ovoid and $\mathcal{L}^{\prime}$ is not a partial spread.

Proof. It is sufficient to show that $\mathcal{P}^{\prime}$ cannot be a partial ovoid. It then follows by duality that $\mathcal{L}^{\prime}$ cannot be a spread. So assume to the contrary that $\mathcal{P}^{\prime}$ is a partial ovoid, this will lead to a contradiction.

Take a point $P \in \mathcal{P}^{\prime}$. Since $\mathcal{P}^{\prime}$ is a partial ovoid, all points collinear with $P$ are not in $\mathcal{P}^{\prime}$. So they need to be covered by at least $q^{2}+q$ different lines from $\mathcal{L}_{D}$. By Lemma 2.9 we now have that $\left|\mathcal{L}_{D}\right|=q^{2}+q$ and $\left|\mathcal{P}_{D}\right|=q^{2}-q+1 \geq\left|\mathcal{P}^{\prime}\right|$.

Now let $\eta$ be the number of lines that are blocked by points of $\mathcal{P}_{D} \backslash \mathcal{P}^{\prime}$, but are not in $\mathcal{L}_{D}$ and are not blocked by a point of $\mathcal{P}^{\prime}$. Then we can count the total number of lines in $\mathrm{GQ}(q, q)$ :

$$
q^{3}+q^{2}+q+1=\left|\mathcal{L}_{D}\right|+(q+1)\left|\mathcal{P}^{\prime}\right|+\eta .
$$

Note that $\eta \leq\left(q^{2}-q+1-\left|\mathcal{P}^{\prime}\right|\right) q$, since each point of $\mathcal{P}_{D} \backslash \mathcal{P}^{\prime}$ is covered at least once by $\mathcal{L}_{D}$, so it contributes at most $q$ lines to $\eta$. Remember that $\left|\mathcal{L}_{D}\right|=q^{2}+q$. We now find that:

$$
q^{3}+q^{2}+q+1 \leq q^{2}+q+(q+1)\left|\mathcal{P}^{\prime}\right|+\left(q^{2}-q+1-\left|\mathcal{P}^{\prime}\right|\right) q,
$$

which implies that $\left|\mathcal{P}^{\prime}\right| \geq q^{2}-q+1$. This means that $\mathcal{P}^{\prime}=\mathcal{P}_{D}$. So all points of the dominating set are uncovered. 
Since $\left|\mathcal{L}_{D}\right|>q^{2}+1$, the set of lines $\mathcal{L}_{D}$ cannot form a partial spread, meaning some of these lines must intersect. Assume there exist three lines $\ell_{1}, \ell_{2}, \ell_{3} \in \mathcal{L}_{D}$ such that $\ell_{1}$ intersects both $\ell_{2}$ and $\ell_{3}$, in different points. Each point $Q \in \mathcal{P}_{D}$ can be projected onto $\ell_{1}$. The projection point needs to be different from the points where $\ell_{2}$ and $\ell_{3}$ intersect $\ell_{1}$. Otherwise, there would not be enough lines in $\mathcal{L}_{D}$ to cover all points collinear to $Q$. Different points from $\mathcal{P}_{D}$ will have different projection lines, since no two points from $\mathcal{P}_{D}$ are collinear. This implies that $\left|\mathcal{P}_{D}\right| \leq(q-1) q=q^{2}-q$, which is a contradiction. From this we can conclude that each line of $\mathcal{L}_{D}$ must cover at least $q$ points which are not covered by any other line of $\mathcal{L}_{D}$.

We can now start counting again:

$$
q^{3}+q^{2}+q+1>\left|\mathcal{P}_{D}\right|+q\left|\mathcal{L}_{D}\right|=q^{2}-q+1+q\left(q^{2}+q\right)=q^{3}+2 q^{2}-q+1,
$$

from which $q<2$ follows, yielding an obvious contradiction. Hence, $\mathcal{P}^{\prime}$ cannot be a partial ovoid.

Note that since $\mathcal{P}^{\prime} \subseteq \mathcal{P}_{D}$, this lemma implies that $\mathcal{P}_{D}$ cannot be a partial ovoid either and, dually, $\mathcal{L}_{D}$ is not a partial spread.

The following two theorems give a characterization for the dominating set constructed in Theorem 2.1, and its dual.

Theorem 4.2. Let $D=\mathcal{P}_{D} \cup \mathcal{L}_{D}$ be a dominating set in $\mathrm{GQ}(q, q)$ of size $2 q^{2}+1$. Let the degree $\mathrm{d}(\ell)$ of a line $\ell$ be the number of points of $\mathcal{P}^{\prime}$ that are incident with $\ell$. If all lines in $\mathrm{GQ}(q, q)$ have $\mathrm{d}(\ell) \in\{0,1, q+1\}$, then $D$ is the dominating set from Theorem 2.1.

Proof. Suppose that every line $\ell$ of the $\operatorname{GQ}(q, q)$ admits $\mathrm{d}(\ell) \in\{0,1, q+1\}$. If there is no line with $\mathrm{d}(\ell)=q+1$, then $\mathcal{P}^{\prime}$ is a partial ovoid, which is not possible according to Lemma 4.1. So there is at least one line with degree $q+1$. Then every point of $\mathcal{P}^{\prime}$ must be contained in a line of degree at least two since either it is contained in a line of degree $q+1$ or it can be projected to one such line, and then the projection line has degree at least two. Since as soon as a line has degree at least two, it is completely contained in $\mathcal{P}^{\prime}$ as a point set, this yields that $\mathcal{P}^{\prime}$ can be obtained as the union of some lines.

Assume there are two non-intersecting lines $\ell$ and $m$ contained (as a set of points) in $\mathcal{P}^{\prime}$. Then each point of $\ell$ can be projected onto $m$. All these projection lines have degree at least two, so they are contained in $\mathcal{P}^{\prime}$ as well (as point sets). But then $\left|\mathcal{P}_{D}\right| \geq(q+1)^{2}>q^{2}+q$, which contradicts Lemma 2.9.

Hence, $\mathcal{P}^{\prime}$ is a set of $k$ lines through a point $P$. Note that $\left|\mathcal{P}^{\prime}\right|=k q+1$, so by Lemma 2.9, $1 \leq k \leq q$. There are $q+1-k$ lines through $P$ that, aside from $P$ itself, do not contain points of $\mathcal{P}^{\prime}$. So all points on these lines, except for $P$, must be covered. This leads to $(q+1-k) q$ lines from $\mathcal{L}_{D}$. These lines of $\mathcal{L}$ cover altogether at most $(q+1-k) q(q+1)$ points. The other lines of $\mathcal{L}_{D}$ can cover at most $q$ points that are not covered yet by these first lines. This leads to the following inequality:

$$
\begin{aligned}
q^{3}+q^{2}+q+1 & \leq k q+1+(q+1-k) q(q+1)+\left(\left|\mathcal{L}_{D}\right|-(q+1-k) q\right) q \\
& =k q+1+(q+1-k) q^{2}+q^{2}+q-k q+\left|\mathcal{L}_{D}\right| q-(q+1-k) q^{2} \\
& =q^{2}+q+1+\left|\mathcal{L}_{D}\right| q
\end{aligned}
$$

hence $\left|\mathcal{L}_{D}\right| \geq q^{2}$. 
The number of lines blocked by the elements of $\mathcal{P}^{\prime}$ is $k q^{2}+q+1$. Consider a point $Q$ in $\mathcal{P}_{D} \backslash \mathcal{P}^{\prime}$. This point needs to be covered at least once by a line of $\mathcal{L}_{D}$. By projecting this point on one of the $k$ lines through $P$, we see that there is at least one line through $Q$ that is already blocked by a point from $\mathcal{P}^{\prime}$. Hence, each point of $\mathcal{P}_{D} \backslash \mathcal{P}^{\prime}$ can block at most $q-1$ lines that are not in $\mathcal{L}_{D}$ and are not blocked by a point of $\mathcal{P}^{\prime}$. So for the size of this set we obtain

$$
\left|\mathcal{P}_{D} \backslash \mathcal{P}^{\prime}\right| \geq \frac{q^{3}+q^{2}+q+1-\left|\mathcal{L}_{D}\right|-\left(k q^{2}+q+1\right)}{q-1}=\frac{q^{3}+q^{2}-k q^{2}-\left|\mathcal{L}_{D}\right|}{q-1} .
$$

Using this inequality and the observation that $\left|\mathcal{L}_{D}\right| \geq q^{2}$, we find for the size of the dominating set $D=\left(\mathcal{P}_{D} \backslash \mathcal{P}^{\prime}\right) \cup \mathcal{P}^{\prime} \cup \mathcal{L}_{D}$ that

$$
\begin{aligned}
|D| & \geq \frac{q^{3}+q^{2}-k q^{2}-\left|\mathcal{L}_{D}\right|}{q-1}+k q+1+\left|\mathcal{L}_{D}\right| \\
& =\frac{q^{3}+q^{2}-k q^{2}}{q-1}+k q+1+\left(1-\frac{1}{q-1}\right)\left|\mathcal{L}_{D}\right| \geq \frac{q^{3}-k q^{2}}{q-1}+k q+1+q^{2} \\
& =q^{2}+(1-k) q+(1-k)+\frac{1-k}{q-1}+k q+1+q^{2} \\
& =2 q^{2}+q+2-k-\frac{k-1}{q-1} .
\end{aligned}
$$

Now assuming $k<q$, we find that $|D|>2 q^{2}+1$, which is a contradiction. So the only possibility left is $k=q$. In this case $\mathcal{P}^{\prime}$ consists of the points on $q$ lines through $P$, and $\left|\mathcal{P}^{\prime}\right|=q^{2}+1$. The number of lines blocked by these points is $q^{3}+q+1$. Since $\left|\mathcal{L}_{D}\right| \geq q^{2}$, all lines not blocked by the points of $\mathcal{P}^{\prime}$ must be in $\mathcal{L}_{D}$. So $D$ is the dominating set constructed in Theorem 2.1.

Dualizing this theorem gives us a characterization for the dual of the construction in Theorem 2.1.

Theorem 4.3. Let $D=\mathcal{P}_{D} \cup \mathcal{L}_{D}$ be a dominating set in $\mathrm{GQ}(q, q)$ of size $2 q^{2}+1$. Let the degree $\mathrm{d}(P)$ of a point $P$ be the number of lines of $\mathcal{L}^{\prime}$ that are incident with $P$. If all points in $\mathrm{GQ}(q, q)$ have $\mathrm{d}(P) \in\{0,1, q+1\}$, then $D$ is the dual dominating set from Theorem 2.1.

We will need the following lemma, which is actually a variation on Lemma 2.5.

Lemma 4.4. Let $D=\mathcal{P}_{D} \cup \mathcal{L}_{D}$ be a dominating set in $\mathrm{GQ}(q, q)$ of size $2 q^{2}+1$. Let $p:=\left|\mathcal{P}_{D}\right|, l:=\left|\mathcal{L}_{D}\right|$, and define $\Delta_{\mathcal{P}}=q^{2}+q-l$ and $\Delta_{\mathcal{L}}=q^{2}+q-p$ as in Lemma 2.8 . Define the degree $\mathrm{d}(\ell)$ of a line $\ell$ as the number of points from $\mathcal{P}_{D}$ incident with $\ell$, and the degree $\mathrm{d}(P)$ of a point $P$ as the number of lines from $\mathcal{L}_{D}$ incident with $P$. Finally, we introduce

$$
\begin{aligned}
c(D)=\sum_{Q \notin \mathcal{P}_{D}}(q+1-\mathrm{d}(Q))(\mathrm{d}(Q)-1)+\sum_{\ell \notin \mathcal{L}_{D}}(q+1-\mathrm{d}(\ell))(\mathrm{d}(\ell)-1) & +\sum_{P \in \mathcal{P}_{D}} \mathrm{~d}(P)+\sum_{\ell \in \mathcal{L}_{D}} \mathrm{~d}(\ell) .
\end{aligned}
$$


Then

$$
\begin{aligned}
& p \geq\left(q^{2}+1-l\right)(q+1)+\frac{1}{q+1}\left(l \Delta_{\mathcal{L}}+c(D)\right), \\
& l \geq\left(q^{2}+1-p\right)(q+1)+\frac{1}{q+1}\left(p \Delta_{\mathcal{P}}+c(D)\right) .
\end{aligned}
$$

Proof. Let $p^{\prime}:=\left|\mathcal{P}^{\prime}\right|$ and $l^{\prime}:=\left|\mathcal{L}^{\prime}\right|$. Note that for a line $\ell, \mathrm{d}(\ell) \geq 1$ iff $\ell \notin \mathcal{L}^{\prime}$. With $p(q+1)$, we count each line $\ell$ blocked by $\mathcal{P}_{D}$ exactly $\mathrm{d}(\ell)$ times, hence the number of lines blocked by $\mathcal{P}_{D}$ is

$$
\begin{aligned}
& p(q+1)-\sum_{\ell \notin \mathcal{L}_{D}}(\mathrm{~d}(\ell)-1)-\sum_{\ell \in \mathcal{L}_{D} \backslash \mathcal{L}^{\prime}}(\mathrm{d}(\ell)-1) \\
& =p(q+1)-\sum_{\ell \notin \mathcal{L}_{D}}(\mathrm{~d}(\ell)-1)-\left(\sum_{\ell \in \mathcal{L}_{D}} \mathrm{~d}(\ell)-l+l^{\prime}\right) .
\end{aligned}
$$

Recall that $l^{\prime}$ equals the number of lines not blocked by $\mathcal{P}_{D}$, hence $l^{\prime}=\left(q^{2}+1\right)(q+1)-$ (4.1). From this it follows that

$$
l=\left(q^{2}+1-p\right)(q+1)+\sum_{\ell \notin \mathcal{L}_{D}}(\mathrm{~d}(\ell)-1)+\sum_{\ell \in \mathcal{L}_{D}} \mathrm{~d}(\ell) .
$$

We will estimate the middle term using

$$
(q+1) \sum_{\ell \notin \mathcal{L}_{D}}(\mathrm{~d}(\ell)-1)=\sum_{\ell \notin \mathcal{L}_{D}} \mathrm{~d}(\ell)(\mathrm{d}(\ell)-1)+\sum_{\ell \notin \mathcal{L}_{D}}(q+1-\mathrm{d}(\ell))(\mathrm{d}(\ell)-1) .
$$

Note that the second sum on the right-hand side is a part of $c(D)$. For the sum $\sum_{\ell \notin \mathcal{L}_{D}} \mathrm{~d}(\ell)(\mathrm{d}(\ell)-1)$ we can find a lower bound as follows.

For $P \in \mathcal{P}_{D}$, let $N^{\prime}(P)$ denote the number of points of $\mathcal{P}^{\prime}$ collinear with $P$. Then

$$
\begin{aligned}
\sum_{\ell \notin \mathcal{L}_{D}} \mathrm{~d}(\ell)(\mathrm{d}(\ell)-1) & =\left|\left\{(P, Q, \ell): \ell \notin \mathcal{L}_{D}, P \in \mathcal{P}_{D}, Q \in \mathcal{P}_{D}, P \sim Q, P Q=\ell\right\}\right| \\
& \geq\left|\left\{(P, Q, \ell): \ell \notin \mathcal{L}_{D}, P \in \mathcal{P}_{D}, Q \in \mathcal{P}^{\prime}, P \sim Q, P Q=\ell\right\}\right| \\
& =\left|\left\{(P, Q): P \in \mathcal{P}_{D}, Q \in \mathcal{P}^{\prime}, P \sim Q\right\}\right|=\sum_{P \in \mathcal{P}_{D}} N^{\prime}(P) .
\end{aligned}
$$

Let $P \in \mathcal{P}_{D}$. Then we have

$$
\begin{gathered}
l \geq \mathrm{d}(P)+\sum_{\substack{Q \sim P \\
P Q \notin \mathcal{L}_{D}}} \mathrm{~d}(Q)=\mathrm{d}(P)+\sum_{\substack{Q \sim P \\
Q \notin \mathcal{P}^{\prime} \\
P Q \notin \mathcal{L}_{D}}} \mathrm{~d}(Q) \\
=\mathrm{d}(P)+(q+1-\mathrm{d}(P)) q-N^{\prime}(P)+\sum_{\substack{Q \sim P \\
Q \notin \mathcal{P}^{\prime} \\
P Q \notin \mathcal{L}_{D}}}(\mathrm{~d}(Q)-1),
\end{gathered}
$$

whence

$$
N^{\prime}(P) \geq \Delta_{\mathcal{P}}-(q-1) \mathrm{d}(P)+\sum_{\substack{Q \sim P \\ Q \notin \mathcal{P}^{\prime} \\ P Q \notin \mathcal{L}_{D}}}(\mathrm{~d}(Q)-1)
$$


With this we find

$$
\begin{aligned}
\sum_{P \in \mathcal{P}_{D}} N^{\prime}(P) & \geq p \Delta_{\mathcal{P}}-(q-1) \sum_{P \in \mathcal{P}_{D}} \mathrm{~d}(P)+\sum_{P \in \mathcal{P}_{D}} \sum_{\substack{Q \sim P \\
Q \notin \mathcal{P}^{\prime} \\
P Q \notin \mathcal{L}_{D}}}(\mathrm{~d}(Q)-1) \\
& \geq p \Delta_{\mathcal{P}}-(q-1) \sum_{P \in \mathcal{P}_{D}} \mathrm{~d}(P)+\sum_{P \in \mathcal{P}_{D}} \sum_{\substack{Q \sim P \\
Q \notin \mathcal{P}_{D} \\
P Q \notin \mathcal{L}_{D}}}(\mathrm{~d}(Q)-1) \\
& =p \Delta_{\mathcal{P}}-(q-1) \sum_{P \in \mathcal{P}_{D}} \mathrm{~d}(P)+\sum_{\substack { Q \notin \mathcal{P}_{D} \\
\begin{subarray}{c}{P \in \mathcal{P}_{D} \\
Q \sim P \\
P Q \notin \mathcal{L}_{D}{ Q \notin \mathcal { P } _ { D } \\
\begin{subarray} { c } { P \in \mathcal { P } _ { D } \\
Q \sim P \\
P Q \notin \mathcal { L } _ { D } } }\end{subarray}}(d(Q)-1) .
\end{aligned}
$$

As for each point $Q \notin \mathcal{P}_{D}$, there are $q+1-\mathrm{d}(Q)$ lines on $Q$ that are not in $\mathcal{L}_{D}$ and each of these must be incident with a point of $\mathcal{P}_{D}$, we find that

$$
\sum_{P \in \mathcal{P}_{D}} N^{\prime}(P) \geq p \Delta_{\mathcal{P}}-(q-1) \sum_{P \in \mathcal{P}_{D}} \mathrm{~d}(P)+\sum_{Q \notin \mathcal{P}_{D}}(q+1-\mathrm{d}(Q))(\mathrm{d}(Q)-1) .
$$

As $\sum_{\ell \in \mathcal{L}_{D}} \mathrm{~d}(\ell)=\sum_{P \in \mathcal{P}_{D}} \mathrm{~d}(P)$, we conclude

$$
\sum_{\ell \notin \mathcal{L}_{D}} \mathrm{~d}(\ell)(\mathrm{d}(\ell)-1) \geq p \Delta_{\mathcal{P}}-(q-1) \sum_{\ell \in \mathcal{L}_{D}} \mathrm{~d}(\ell)+\sum_{Q \notin \mathcal{P}_{D}}(q+1-\mathrm{d}(Q))(\mathrm{d}(Q)-1) .
$$

Together with (4.2) and (4.3), this gives the second desired inequality. The other inequality is showed analogously.

Note that $\mathcal{P}^{\prime}=\mathcal{P}_{D}$ and $\mathcal{L}^{\prime}=\mathcal{L}_{D}$ are equivalent. Also note that if this is the case, then $\sum_{\ell \in \mathcal{L}_{D}} \mathrm{~d}(\ell)=0$. We can now prove the following Theorem, giving a classification of the small dominating sets in $\operatorname{GQ}(q, q)$.

Theorem 4.5. Let $D=\mathcal{P}_{D} \cup \mathcal{L}_{D}$ be a dominating set in $\mathrm{GQ}(q, q)$ with size $|D|=2 q^{2}+1$. Then $D$ is the dominating set from Theorem 2.1 or its dual.

Proof. Define $p=\left|\mathcal{P}_{D}\right|, l=\left|\mathcal{L}_{D}\right|$ and $p^{\prime}=\left|\mathcal{P}^{\prime}\right|$; note that $p+l=2 q^{2}+1$. By duality, we may assume that $p>l+1$ or $p=q^{2}$ (and $l=q^{2}+1$ ). Define the degree $\mathrm{d}(\ell)$ of a line $\ell$ as the number of points from $\mathcal{P}_{D}$ incident with $\ell$, and the degree $\mathrm{d}(P)$ of a point $P$ as the number of lines from $\mathcal{L}_{D}$ incident with $P$. We will find lower bounds on the sums from Lemma 4.4; let $c(D)$ be defined as therein.

Define $\Delta:=\Delta_{\mathcal{P}}=q^{2}+q-l$ as in Lemma 2.8. For any point $P \in \mathcal{P}_{D}$, define the number of neighbors $N(P):=\left|\left\{Q \mid Q \sim P, Q \in \mathcal{P}_{D}\right\}\right|$. We immediately have that $N(P) \geq \Delta$ if $P \in \mathcal{P}^{\prime}$. If $l=q^{2}+1$, then $\Delta=q-1$. If $p>l+1$, then $q^{2}-q+1 \leq l<q^{2}$, by Lemma 2.9 . In both cases, we have that $\Delta \not \equiv 0(\bmod q)$. We now consider two types of points in $\mathcal{P}^{\prime}$ and their contributions to $c(D)$.

- Type 1: $P$ is incident with at least one line $e$ with $2 \leq \mathrm{d}(e) \leq q$.

Since $P$ is a point from $\mathcal{P}^{\prime}$, the line $e$ is not in $\mathcal{L}_{D}$. So this line $e$ contributes at least $q-1$ to $\sum_{\ell \notin \mathcal{L}_{D}}(q+1-\mathrm{d}(\ell))(\mathrm{d}(\ell)-1)$. Note that on this line there are at most 
$q$ points of Type 1 . Assume there are $k$ points of Type 1 , then we find the following lower bound:

$$
\sum_{\ell \notin \mathcal{L}_{D}}(q+1-\mathrm{d}(\ell))(\mathrm{d}(\ell)-1) \geq k \frac{q-1}{q} .
$$

- Type 2: All lines through $P$ have degree 1 or $q+1$.

If $\Delta=N(P)$, then there are exactly $\Delta$ points in $\mathcal{P}_{D}$ collinear with $P$. But $\Delta \neq \equiv$ $(\bmod q)$, so then there must be at least one line $\ell$ through $P$ with degree $2 \leq \mathrm{d}(\ell) \leq$ $q$. Then $P$ would be a point of Type 1 . So we have $N(P)>\Delta$.

Denote by $x_{i}(P):=\left|\left\{R \mid R \notin \mathcal{P}_{D}, R \sim P, \mathrm{~d}(R)=i\right\}\right|$, for $i=1, \ldots, q$. Note that, as $P \in \mathcal{P}^{\prime}$ there are no points collinear with $P$ with degree $q+1$.

Each point $R \notin \mathcal{P}_{D}$, with degree $1 \leq i \leq q$ contributes $(q+1-i)(i-1)$ to the sum $\sum_{Q \notin \mathcal{P}_{D}}(q+1-\mathrm{d}(Q))(\mathrm{d}(Q)-1)$. Such a point is collinear with at most $q+1-i$ points of Type 2 , since a line through a point of Type 2 is not in $\mathcal{L}_{D}$, and either contains no other points of $\mathcal{P}_{D}$ or contains only points of $\mathcal{P}_{D}$. So the contribution of $P$ to $\sum_{Q \notin \mathcal{P}_{D}}(q+1-\mathrm{d}(Q))(\mathrm{d}(Q)-1)$ is at least

$$
\sum_{i=1}^{q} x_{i} \frac{(q+1-i)(i-1)}{q+1-i}=\sum_{i=1}^{q} x_{i}(i-1) .
$$

Now we show that the contribution is strictly positive for each point of Type 2. So assume this is not the case for a point $P \in \mathcal{P}^{\prime}$ of Type 2, so each point $Q \notin \mathcal{P}_{D}$ collinear with $P$ has degree $\mathrm{d}(Q)=1$. Since each line through $P$ has either degree 1 or $q+1$, there must be $\lambda$ lines through $P$ which contain all points collinear with it from $\mathcal{P}$, for some $1 \leq \lambda \leq q+1(\lambda>0$ as $N(P)>0)$. So we already have $\lambda q+1$ points in the dominating set.

On each of the other $q+1-\lambda$ lines through $P$ there are $q$ points with degree 1 . This gives us $q(q+1-\lambda)$ lines in the dominating set. Through each of these points $\notin \mathcal{P}_{D}$, collinear with $P$ there are $q-1$ lines which are not in the dominating set and are not blocked yet. Say there are $x$ points in $D$, which are not collinear with $P$ and different from $P$ itself. Each of these points can block at most $q+1-\lambda$ lines which are not yet blocked. From this follows $(q-1) q(q+1-\lambda) \leq x(q+1-\lambda)$, hence $x \geq q^{2}-q$. Since $2 q^{2}+1=|D| \geq \lambda q+1+x+(q+1-\lambda) q=q^{2}+q+1+x$, we have $x=q^{2}-q$. As $q^{2}+q \geq\left|\mathcal{P}_{D}\right|=x+\lambda q+1=q^{2}+(\lambda-1) q+1$, we have $\lambda=1$ and $\left|\mathcal{P}_{D}\right|=q^{2}+1$, contrary to our assumptions.

From this follows that we may assume that for each point $P$ of Type 2 we have $\sum_{i=1}^{q} x_{i}(i-1) \geq 1$. Note that there are $p^{\prime}-k$ points of Type 2 . This gives us the following inequality:

$$
\sum_{Q \notin \mathcal{P}_{D}}(q+1-\mathrm{d}(Q))(\mathrm{d}(Q)-1) \geq p^{\prime}-k .
$$

Note that $\mathrm{d}(P) \geq 1$ for each $P \in \mathcal{P}_{D} \backslash \mathcal{P}^{\prime}$, so $\sum_{\ell \in \mathcal{L}_{D}} \mathrm{~d}(\ell)=\sum_{P \in \mathcal{P}_{D}} \mathrm{~d}(P) \geq p-p^{\prime}$. 
Combining this with (4.4) and (4.5), and with $k \leq p^{\prime} \leq p$ and $p \geq q^{2}$, we get the following:

$$
\begin{aligned}
c(D) & =\sum_{\ell \notin \mathcal{L}_{D}}(q+1-\mathrm{d}(\ell))(\mathrm{d}(\ell)-1) \\
& +\sum_{Q \notin \mathcal{P}_{D}}(q+1-\mathrm{d}(Q))(\mathrm{d}(Q)-1)+2 \sum_{P \in \mathcal{P}_{D}} \mathrm{~d}(P) \\
& \geq k \frac{q-1}{q}+p^{\prime}-k+2\left(p-p^{\prime}\right) \geq k \frac{q-1}{q}+p-k \\
& \geq p-\frac{k}{q} \geq p \frac{q-1}{q} \geq q^{2}-q .
\end{aligned}
$$

Thus, according to Lemma 4.4, we find

$$
\begin{aligned}
& p \geq\left(q^{2}+1-l\right)(q+1)+\frac{1}{q+1}\left(l \Delta_{l}+q^{2}-q\right), \\
& l \geq\left(q^{2}+1-p\right)(q+1)+\frac{1}{q+1}\left(p \Delta_{p}+q^{2}-q\right) .
\end{aligned}
$$

Now using $2 q^{2}+1=|D|=p+l, \Delta_{\mathcal{P}}=q^{2}+q-l, \Delta_{\mathcal{L}}=q^{2}+q-p$, and that $p l \leq q^{2}\left(q^{2}+1\right)$, we calculate the sum of these two inequalities:

$$
\begin{aligned}
2 q^{2}+1 & \geq q+1+\frac{p\left(q^{2}+q-l\right)+l\left(q^{2}+q-p\right)+2\left(q^{2}-q\right)}{q+1} \\
& =q+1+\frac{(p+l) q(q+1)-2 p l+2\left(q^{2}-q\right)}{q+1} \\
& =q+1+q\left(2 q^{2}+1\right)+\frac{-2 p l+2\left(q^{2}-q\right)}{q+1} \\
& \geq 2 q^{3}+2 q+1+\frac{-2 q^{4}-2 q^{2}+2 q^{2}-2 q}{q+1} \\
& \geq 2 q^{3}+2 q+1-2 q \frac{q^{3}+1}{q+1} \\
& \geq 2 q^{3}+2 q+1-2 q\left(q^{2}-q+1\right)=2 q^{2}+1 .
\end{aligned}
$$

So we see that we actually reach equality. This means that all the estimates we used during our countings were exact, hence we have $k=p^{\prime}=p$. As $\mathcal{P}_{D}=\mathcal{P}^{\prime}$, every point $P \in \mathcal{P}_{D}$ has $\mathrm{d}(P)=0$. Equality with zero in (4.5) yields that for all $Q \notin \mathcal{P}_{D}$ we have $\mathrm{d}(Q)=1$ or $\mathrm{d}(Q)=q+1$. By Theorem 4.3, $D$ is the dual of the dominating set from Theorem 2.1.

\section{Conclusion, remarks and open problems}

The main results of this paper can be summarized as follows.

\section{Theorem 5.1.}

- The domination number of $\mathrm{GQ}(s, t)$ is at most $2 s t+1$.

- The domination number of $\mathrm{GQ}(q, q)$ equals $2 q^{2}+1$.

- A dominating set of $\operatorname{GQ}(q, q)$ of size $2 q^{2}+1$ is one of the two examples seen in Figure 1. 
- The domination number of $\mathrm{GQ}(q-1, q+1)$ and $\mathrm{GQ}(q+1, q-1)$ equals $2 q^{2}-1$.

Let us outline some possible areas of further investigation in this topic. For the other classical parameters, the calculations in Lemma 3.1 fail in general. Is the bound $2 s t+1$ for $\operatorname{GQ}(s, t)$ still sharp for general $s$ and $t$ ? It would be also interesting to answer this question for the classical generalized quadrangles. We have checked by computer, using a simple linear integer programming model and Gurobi [7], for all classical GQs of order $(3,9),(4,8),(4,16)$ as given on Moorhouse's webpage [12], and we have found the bound sharp.

In $W(q)$, or $Q(4, q)$, if $q$ is even, there exists an ovoid and a spread as well, giving rise to a dominating set of size $2 q^{2}+2$. This implies that there is no general stability phenomenon for smallest dominating sets in GQs, unlike in the case of generalized triangles (projective planes; see [11]); that is, the size of minimal examples (with respect to containment) may be arbitrarily close. However, the structure of the mentioned dominating sets are immensely dissimilar. Is it true that minimal dominating sets of size $2 q^{2}+2$ of a $\mathrm{GQ}(q, q)$ (or, more specifically, $W(q)$ ) are the union of an ovoid and a spread? What size does the next smallest minimal example for a dominating set in $\operatorname{GQ}(q, q)$ have?

Dominating sets of a graph $G=(V, E)$ may be also viewed as a set $D$ of vertices such that $V \backslash D$ induces a subgraph of $G$ such that every vertex has degree at least one smaller than originally. If equality holds for every vertex of $V \backslash D$, then $D$ is called a perfect dominating set. More generally, if we replace 'at least one smaller' by 'at least $t$ smaller', we talk about $t$-fold dominating sets and perfect $t$-fold dominating sets. Perfect $t$ fold dominating sets of the incidence graphs of projective planes, generalized quadrangles and generalized hexagons have already been studied in order to produce upper bounds on the order of some particular cage graphs (see [9] for an overview). In [5], perfect $t$-fold dominating sets of the incidence graph of the desarguesian projective plane $\operatorname{PG}(2, q)$ are completely described for small enough $t$, while the characterization of small dominating sets of projective planes can be found in [11]. In the case $t=1$, describing smallest dominating and perfect dominating sets is quite easy, unlike in the here discussed case of generalized quadrangles; see also [2] for results on perfect (1-fold) dominating sets of $Q(4, q)$. It would be also interesting to study $t$-fold (ordinary and perfect) dominating sets of generalized quadrangles, $t \geq 2$. Also, as a counterpart of $t$-fold dominating sets, finding a (preferably large) subset $D$ of the incidence graph such that each vertex not in $D$ has at most $t$ neighbors in $D$ would be also interesting, as its complement induces a subgraph of high minimum degree. Such subsets, asides being interesting in themselves, also may find their applications in different topics as they do when the host graph is the incidence graph of a projective plane; see $[1,10]$.

\section{ORCID iDs}

Tamás Héger (iD https://orcid.org/0000-0002-9586-966X

Lisa Hernandez Lucas (iD https://orcid.org/0000-0002-1356-5970

\section{References}

[1] G. Araujo-Pardo and C. Balbuena, Constructions of small regular bipartite graphs of girth 6, Networks 57 (2011), 121-127, doi:10.1002/net.20392.

[2] L. Beukemann and K. Metsch, Regular graphs constructed from the classical generalized quadrangle $Q(4, q)$, J. Combin. Des. 19 (2011), 70-83, doi:10.1002/jcd.20266. 
[3] E. J. Cockayne and S. T. Hedetniemi, Towards a theory of domination in graphs, Networks 7 (1977), 247-261, doi:10.1002/net.3230070305.

[4] A. Gács and T. Héger, On geometric constructions of $(k, g)$-graphs, Contrib. Discrete Math. 3 (2008), 63-80, doi:10.11575/cdm.v3i1.61998.

[5] A. Gács, T. Héger and Zs. Weiner, On regular graphs of girth six arising from projective planes, European J. Combin. 34 (2013), 285-296, doi:10.1016/j.ejc.2012.07.005.

[6] F. Goldberg, D. Rajendraprasad and R. Mathew, Domination in designs, arXiv: 1405.3436 [math. CO].

[7] Gurobi Optimization, LLC, Gurobi Optimizer (Version 8.1), 2020, https://www . gurobi.com.

[8] S. T. Hedetniemi and R. C. Laskar, Bibliography on domination in graphs and some basic definitions of domination parameters, Discrete Math. 86 (1990), 257-277, doi:10.1016/ 0012-365x(90)90365-o.

[9] T. Héger, Some graph theoretic aspects of finite geometries, Ph.D. thesis, ELTE Eötvös Loránd University, 2013.

[10] T. Héger and S. Mattheus, Bipartite Ramsey numbers for the four-cycle versus stars emerging from projective planes, 2020, manuscript.

[11] T. Héger and Z. L. Nagy, Dominating sets in projective planes, J. Combin. Des. 25 (2017), 293-309, doi:10.1002/jcd.21527.

[12] E. Moorhouse, Generalised Polygons of Small Order, 2003, http://ericmoorhouse. org/pub/genpoly/.

[13] S. E. Payne and J. A. Thas, Finite Generalized Quadrangles, EMS Series of Lectures in Mathematics, European Mathematical Society (EMS), Zürich, 2nd edition, 2009, doi:10.4171/066.

[14] J. Tits, Sur la trialité et certains groupes qui s'en déduisent, Inst. Hautes Études Sci. Publ. Math. 2 (1959), 13-60, http: / / www . numdam.org/item? id=PMIHES_1959_2__13_0. 